\title{
ANNALES
}

UNIVERSITATIS MARIAE CURIE-SKŁODOWSKA

LUBLIN - POLONIA

VOL. LXV, NO. 2, 2011

SECTIO A

$179-190$

JÓZEF SICIAK

\section{Some gap power series in multidimensional setting}

\author{
Professor Jan Krzyż in memoriam
}

Abstract. We study extensions of classical theorems on gap power series of a complex variable to the multidimensional case.

\section{Power series with Ostrowski gaps. Let}

$$
f(z)=\sum_{0}^{\infty} Q_{j}(z), \quad \text { where } \quad Q_{j}(z)=\sum_{|\alpha|=j} a_{\alpha} z^{\alpha}, \quad \alpha \in \mathbb{Z}_{+}^{N},
$$

be a power series in $\mathbb{C}^{N}$, i.e. a series of homogeneous polynomials $Q_{j}$ of $N$ complex variables of degree $j$.

The set $\mathcal{D}$ given by the formula $\mathcal{D}:=\left\{a \in \mathbb{C}^{N}\right.$; the sequence (1.1) is convergent in a neighborhood of $a\}$ is called a domain of convergence of (1.1).

It is known that

$$
\mathcal{D}=\left\{z \in \mathbb{C}^{N} ; \psi^{*}(z)<1\right\},
$$

where

$$
\psi(z):=\limsup _{j \rightarrow \infty} \sqrt[j]{\left|Q_{j}(z)\right|}
$$

and $\psi^{*}$ denotes the upper semicontinuous regularization of $\psi$.

2000 Mathematics Subject Classification. 30B10, 30B30, 30B40, 32A05, 32A07, 32A10, $32 \mathrm{D} 15$.

Key words and phrases. Plurisubharmonic functions, negligible sets in $\mathbb{C}^{N}$, power series, lacunary power series, multiple power series. 
If $\psi^{*}$ is finite, then it is plurisubharmonic and absolutely homogeneous (i.e. $\left.\psi^{*}(\lambda z)=|\lambda| \psi^{*}(z), \lambda \in \mathbb{C}, z \in \mathbb{C}^{N}\right)$. Therefore, the domain of convergence $\mathcal{D}$ is either empty, or it is a balanced (i.e. $\lambda z \in \mathcal{D}$ for all $\lambda \in \mathbb{C}$ with $|\lambda| \leq 1$ and $z \in \mathcal{D}$ ) domain of holomorphy. Every balanced domain of holomorphy is a domain of convergence of a series (1.1).

For every balanced domain $D$ in $\mathbb{C}^{N}$ there is a unique nonnegative function $h$ (so-called Minkowski functional of $D$ ) such that $h(\lambda z)=|\lambda| h(z)$ for all $\lambda \in \mathbb{C}$ and $z \in \mathbb{C}^{N}$, and $D=\left\{z \in \mathbb{C}^{N} ; h(z)<1\right\}$. In particular, if $\mathcal{D}$ is a domain of convergence of (1.1), then $h(z) \equiv \psi^{*}(z)$.

It is known that a balanced domain in $\mathbb{C}^{N}$ is a domain of holomorphy if and only if its Minkowski functional $h$ is an absolutely homogeneous plurisubharmonic function.

The number

$$
\rho:=1 / \limsup _{j \rightarrow \infty} \sqrt[j]{\left\|Q_{j}\right\|_{\mathbb{B}}}
$$

where $\mathbb{B}:=\left\{z \in \mathbb{C}^{N} ;\|z\| \leq 1\right\}$, is called a radius of convergence of series (1.1) (with respect to a given norm $\|\cdot\|$ ).

If $N=1$, then $\psi(z)=\frac{|z|}{\rho}$ and $\mathcal{D}=\rho \mathbb{B}$. If $N \geq 2$, then $\rho \mathbb{B} \subset \mathcal{D}$ but, in general, $\mathcal{D} \neq \rho \mathbb{B}$.

Series (1.1) is normally geometrically convergent in $\mathcal{D}$, i.e.

$$
\limsup _{j \rightarrow \infty} \sqrt[j]{\left\|Q_{j}\right\|_{K}}<1, \quad \limsup _{n \rightarrow \infty} \sqrt[n]{\left\|f-s_{n}\right\|_{K}}<1,
$$

for all compact sets $K \subset \mathcal{D}$, where $s_{n}:=Q_{o}+\cdots+Q_{n}$ is the $n$th partial sum of (1.1).

Definition 1.1. We say that a function $f$ holomorphic in a neighborhood of a point $z^{o} \in \mathbb{C}^{N}$ possesses at the point $z^{o}$ Ostrowski's gaps $\left(m_{k}, n_{k}\right]$, if

$1^{o} . m_{k}, n_{k}$ are natural numbers such that $m_{k}<n_{k}<m_{k+1}(k \geq 1)$, $\frac{n_{k}}{m_{k}} \rightarrow \infty$ as $k \rightarrow \infty$

$2^{o} . \lim _{j \rightarrow \infty, j \in I} \sqrt[j]{\left\|Q_{j}\right\|_{\mathbb{B}}}=0$, where $\mathbb{B}$ is the unit ball in $\mathbb{C}^{N}$,

$$
Q_{j}(z) \equiv Q_{j}^{\left(f, z^{o}\right)}(z):=\sum_{|\alpha|=j} \frac{f^{(\alpha)}\left(z^{o}\right)}{\alpha !} z^{\alpha}=\frac{1}{j !}\left(\frac{d}{d \lambda}\right)^{j} f\left(z^{o}+\lambda z\right)_{\mid \lambda=0},
$$

and $I:=\bigcup_{k=1}^{\infty}\left(m_{k}, n_{k}\right],\left(m_{k}, n_{k}\right]$ denoting the set of integers $j$ with $m_{k}<$ $j \leq n_{k}$.

Observe that $f_{o}(z):=\sum_{j \in I} Q_{j}\left(z-z^{o}\right)$ is an entire function such that the function $g:=f-f_{o}$ possesses Ostrowski's gaps $\left(m_{k}, n_{k}\right]$ at $z^{o}$ with $Q_{j}^{\left(g, z^{\circ}\right)}=0$ for $m_{k}<j \leq n_{k}, k \geq 1$. Hence, a holomorphic function $f$ possesses Ostrowski's gaps $\left(m_{k}, n_{k}\right]$ at a point $z^{o}$ if and only if there exists an entire function $f_{o}$ such that $Q_{j}^{\left(f-f_{o}, z^{\circ}\right)}=0$ for $m_{k}<j \leq n_{k}, k \geq 1$. 
Moreover, the maximal domain of existence $G=G_{f}$ of $f$ is identical with the maximal domain of existence of $f-f_{o}$.

Definition 1.2. We say that a function $f$ holomorphic in a neighborhood of a point $z^{o}$ possesses Ostrowski's gaps relative to a sequence of positive integers $\left\{n_{k}\right\}$, if $\left\{n_{k}\right\}$ is increasing and there exists a sequence of positive real numbers $\left\{q_{k}\right\}$ such that $q_{k} \rightarrow 0$ as $k \rightarrow \infty$ and $\lim _{j \rightarrow \infty, j \in I} \sqrt[j]{\left\|Q_{j}\right\|_{\mathbb{B}}}=0$, where $I:=\bigcup_{k=1}^{\infty}\left(\left\lfloor q_{k} n_{k}\right\rfloor, n_{k}\right\rfloor$.

A function $f$ possesses Ostrowski's gaps according to Definition 1.1 if and only if $f$ possesses Ostrowski's gaps according to Definition 1.2.

Indeed, if the conditions of Definition 1.1 are satisfied, then it is sufficient to put $q_{k}:=m_{k} / n_{k}$.

If the conditions of Definition 1.2 are satisfied, consider two cases. If $m:=\liminf _{k \rightarrow \infty} q_{k} n_{k}$ is finite, then the function $f$ is entire, so that $f$ has Ostrowski's gaps $\left(m_{k}, n_{k}\right]$ according to Definition 1 for any sequence $m_{k}, n_{k}$ satisfying $1^{\circ}$.

If $\liminf _{k \rightarrow \infty} q_{k} n_{k}=\infty$, then $f$ possesses Ostrowski's gaps $\left(\left\lfloor q_{k_{l}} n_{k_{l}}\right\rfloor, n_{k_{l}}\right\rfloor$ for a suitable chosen increasing subsequence $k_{l}$ of positive integers.

We say that a compact subset $K$ of $\mathbb{C}^{N}$ is polynomially convex if $K$ is identical with its polynomially convex hull $\hat{K}:=\left\{a \in \mathbb{C}^{N} ;|P(a)| \leq\|P\|_{K}\right.$ for every polynomial $P$ of $N$ complex variables $\}$. We say that an open set $\Omega$ in $\mathbb{C}^{N}$ is polynomially convex, if for every compact subset $K$ of $\Omega$ the polynomially convex hull $\hat{K}$ of $K$ is contained in $\Omega$.

The following theorem is known (see [7]). It is a multidimensional version of the classical Ostrowski's Theorem (see Theorem 3.1.1 in [1]).

Theorem 1. If a holomorphic function $f$ possesses Ostrowski's gaps $\left(m_{k}, n_{k}\right]$ at a point $z^{o} \in \mathbb{C}^{N}$, then the maximal domain of existence $G=G_{f}$ of $f$ is one-sheeted and polynomially convex. Moreover, for every compact subset $K$ of $G$ we have

$$
\limsup _{k \rightarrow \infty}\left\|f-s_{n_{k}}\right\|_{K}^{1 / n_{k}}<1
$$

where

$$
s_{n}(z) \equiv s_{n}^{\left(f, z^{o}\right)}(z)=\sum_{j=0}^{n} Q_{j}^{\left(f, z^{o}\right)}\left(z-z^{o}\right)
$$

is the nth partial sum of the Taylor series development of $f$ around $z^{\circ}$.

Corollary 1.1. If

$$
f\left(z^{o}+z\right)=\sum_{k=1}^{\infty} Q_{m_{k}}^{\left(f, z_{o}\right)}(z),
$$

where $m_{k} / m_{k+1} \rightarrow 0$ as $k \rightarrow \infty$, then $Q_{j}^{\left(f, z^{o}\right)}=0$ for $j \notin\left\{m_{k}\right\}$ so that $f$ has Ostrowski's gaps $\left(m_{k}, n_{k}\right]$ with $n_{k}:=m_{k+1}-1$. Therefore, the maximal 
domain of existence $G_{f}$ of $f$ is identical with the domain of convergence $\mathcal{D}_{f}$ of the Taylor series development of $f$ around $z^{o}$, i.e.

$$
G_{f}=\mathcal{D}_{f}:=\left\{z \in \mathbb{C}^{N}: \psi^{*}\left(z-z^{o}\right)<1\right\},
$$

where $\psi(z):=\lim \sup _{k \rightarrow \infty} \sqrt[m]{\left|Q_{m_{k}}^{\left(f, z^{o}\right)}(z)\right|}$.

The following result gives an $N$-dimensional version of W. Luh's Theorem 1 in [4]. In particular, it says that if a function $f$ holomorphic in a domain $G$ in $\mathbb{C}^{N}$ possesses Ostrowski's gaps at some point $z^{o} \in G$, then $f$ possesses the same property at every other point $a$ of the maximal domain of existence of $f$.

Theorem 2. Let $f$ possess Ostrowski's gaps $\left(m_{k}, n_{k}\right]$ at a point $z^{o} \in \mathbb{C}^{N}$. Then

$1^{o}$. f possesses Ostrowski's gaps $\left(m_{k_{l}},\left\lceil\frac{n_{k_{l}}}{l}\right\rceil\right]$ at every point $a \in G_{f}$, where the sequence of natural numbers $\left\{k_{l}\right\}$ (independent of a) is chosen in such a way that $n_{k_{l}} \geq m_{k_{l}} l^{2}$ and $\left\lceil\frac{n_{k_{l}}}{l}\right\rceil<m_{k_{l+1}}$ for $l \geq 1$;

$2^{o}$. If $Q_{j}^{\left(f, z^{o}\right)}=0$ for $m_{k}<j \leq n_{k}, k \geq 1^{1}$, then the sequence $\left\{s_{m_{k}}^{\left(f, z^{o}\right)}-\right.$ $\left.s_{m_{k}}^{(f, a)}\right\}$ converges to zero normally with order $n_{k}$ on $\mathbb{C}^{N}$, i.e.

$$
\limsup _{k \rightarrow \infty}\left\|s_{m_{k}}^{\left(f, z^{o}\right)}-s_{m_{k}}^{(f, a)}\right\|_{K}^{1 / n_{k}}<1
$$

for every compact set $K \subset \mathbb{C}^{N}$.

By $2^{\circ}$ and Theorem 1 we get the following:

Corollary 1.2. If $f$ possesses ordinary Ostrowski's gaps $\left(m_{k}, n_{k}\right]$ at a fixed point $z^{o} \in G$, then

$$
\limsup _{k \rightarrow \infty} \sqrt[n k]{\left\|f-s_{m_{k}}^{(f, a)}\right\|_{K}}<1
$$

for every point $a \in G_{f}$ and every compact subset $K$ of $G_{f}$.

Proof of Theorem 2. $1^{o}$. Without loss of generality we may assume that $z^{o}=0$ and

$$
Q_{j}^{\left(f, z^{\circ}\right)}=0, \quad m_{k}<j \leq n_{k}, \quad k \geq 1 .
$$

Given a fixed point $a \in G_{f}$, we have

$$
Q_{j}^{(f, a)}(z)=\frac{1}{2 \pi i} \int_{|\lambda|=r} \frac{f(a+\lambda z)-s_{n_{k}}(a+\lambda z)}{\lambda^{j+1}} d \lambda
$$

\footnotetext{
${ }^{1}$ In such a case we say that $f$ possesses ordinary Ostrowski's gaps at $z^{o}$
} 
$\|z\| \leq 1, j>m_{k}, k \geq 1$, where $s_{n_{k}}=s_{n_{k}}^{\left(f, z_{o}\right)}$ (Observe that $s_{n_{k}}$ is a polynomial of degree at most $\left.m_{k}\right)$, and $0<r<\min \left(\operatorname{dist}\left(a, \partial G_{f}\right)\right.$, $\left.\operatorname{dist}\left(z^{o}, \partial G_{f}\right)\right)$. By Theorem 1 there exist $M>1$ and $0<\theta<1$ such that

$$
\left\|f-s_{n_{k}}\right\|_{\mathbb{B}(a, r)} \leq M \theta^{n_{k}}, \quad k \geq 1 .
$$

Therefore, by Cauchy inequalities,

$$
\left\|Q_{j}^{(f, a)}\right\|_{\mathbb{B}} \leq \frac{M}{r^{j}} \theta^{n_{k}}, \quad j>m_{k}, \quad k \geq 1 .
$$

Let $\left\{k_{l}\right\}$ be an increasing sequence of natural numbers such that

$$
m_{k_{l+1}}>\left\lceil\frac{n_{k_{l}}}{l}\right\rceil, \quad \frac{n_{k_{l}}}{m_{k_{l}}} \geq l^{2}, \quad l \geq 1
$$

By (1.8) we get

$$
\left\|Q_{j}^{(f, a)}\right\|_{\mathbb{B}}^{1 / j} \leq \frac{M}{r} \theta^{n_{k_{l}} / j} \leq \frac{M}{l} \theta^{l}, \quad m_{k_{l}}<j \leq\left\lceil\frac{n_{k_{l}}}{l}\right\rceil, \quad l \geq 1 .
$$

The choice of the sequence $\left\{k_{l}\right\}$ does not depend on $a \in G_{f}$. Therefore, $f$ possesses Ostrowski's gaps $\left(m_{k_{l}},\left\lceil\frac{n_{k_{l}}}{l}\right\rceil\right]$ at every point $a$ of $G_{f}$ (according to Definition 1.1). The proof of the case $1^{o}$ is ended.

$2^{o}$. Observe that for $\|z-a\| \leq \frac{1}{2} r$ we have

$$
\left|f(z)-s_{m_{k}}^{(f, a)}(z)\right|=\sum_{m_{k}+1}^{\infty}\left|Q_{j}^{(f, a)}(z-a)\right| \leq \sum_{m_{k}+1}^{\infty}\left\|Q_{j}^{(f, a)}\right\|_{\mathbb{B}}\left(\frac{r}{2}\right)^{j},
$$

which by (1.8) gives

$$
\left|f(z)-s_{m_{k}}^{(f, a)}(z)\right| \leq \sum_{p_{k}+1}^{\infty} 2^{-j} M \theta^{n_{k}} \leq M \theta^{n_{k}}, \quad k \geq 1, \quad\|z-a\| \leq \frac{r}{2} .
$$

By (1.7) and (1.9) we get

$$
\left\|s_{m_{k}}^{\left(f, z^{o}\right)}-s_{m_{k}}^{(f, a)}\right\|_{\mathbb{B}\left(a, \frac{1}{2} r\right)} \leq 2 M \theta^{n_{k}}, \quad k \geq 1
$$

Observe that for $z \in \mathbb{C}^{N}$

$$
\begin{aligned}
\left|s_{n}^{\left(f, z^{o}\right)}(z)\right| & \leq \sum_{j=0}^{n}\left\|Q_{j}^{\left(f, z^{o}\right)}\right\|_{\mathbb{B}}\left\|z-z^{o}\right\|^{j} \leq \sum_{0}^{n} \frac{\|f\|_{\mathbb{B}\left(z^{o}, r\right)}}{r^{j}}\left\|z-z^{o}\right\|^{j} \\
& \leq(n+1)\|f\|_{\mathbb{B}\left(z^{o}, r\right)}\left(1+\frac{\|z\|+\left\|z_{o}\right\|}{r}\right)^{n} .
\end{aligned}
$$

Put $M:=\|f\|_{\mathbb{B}\left(z^{o}, r\right) \cup \mathbb{B}(a, r)}$ and $c:=\max \left\{\left\|z^{o}\right\|,\|a\|\right\}$. Then for $z \in \mathbb{C}^{N}$

$$
\begin{aligned}
u_{k}(z) & :=\frac{1}{n_{k}} \log \left|s_{m_{k}}^{\left(f, z^{o}\right)}(z)-s_{m_{k}}^{(f, a)}(z)\right| \\
& \leq \frac{1}{n_{k}} \log \left[2 M\left(m_{k}+1\right)\right]+\frac{m_{k}}{n_{k}} \log \left(1+\frac{\|z\|+\|c\|}{r}\right) .
\end{aligned}
$$


It follows that the sequence of plurisubharmonic functions $\left\{u_{k}\right\}$ is locally uniformly upper bounded in $\mathbb{C}^{N}$, and

$$
u(z):=\limsup _{k \rightarrow \infty} u_{k}(z) \leq 0, \quad z \in \mathbb{C}^{n} .
$$

Therefore, the plurisubhamonic function $u^{*}=$ const.

By $(1.10) u_{k}(z) \leq \frac{1}{n_{k}} \log 2 M+\log \theta$ for $z \in \mathbb{B}(a, r), k \geq 1$. Hence $u^{*} \leq \log \theta$ in $\mathbb{C}^{N}$ which ends the proof of $2^{\circ}$.

2. E. Fabry's Theorem. Now we shall present a multidimensional version of E. Fabry's Theorem (Theorem 2.2.1 in [1]). Let $f$ be a function of $\mathrm{N}$ complex variables holomorphic in a neighborhood of 0 with a gap Taylor series development

$$
f(z)=\sum_{k=1}^{\infty} Q_{m_{k}}(z), \quad m_{k}<m_{k+1} .
$$

Put $\psi(z):=\lim \sup _{k \rightarrow \infty} \sqrt[m_{k}]{\left|Q_{m_{k}}(z)\right|}, h(z):=\psi^{*}(z)$. It is known that $\mathcal{D}:=\left\{z \in \mathbb{C}^{N} ; h(z)<1\right\}=\left\{a \in \mathbb{C}^{N}\right.$; series (2.1) is convergent in a neighborhood of $a\}$ is a domain of convergence of (2.1).

Theorem 3. If $\lim _{k \rightarrow \infty} \frac{k}{m_{k}}=0$, then the domain of convergence $\mathcal{D}$ of the series (2.1) is identical with the maximal domain of existence $G_{f}$ of $f$.

Proof. Without loss of generality we may assume that $\mathcal{D} \neq \mathbb{C}^{N}$.

Due to Fabry we know that Theorem 3 is true for $N=1$. It is also well known (by Bedford-Taylor Theorem on negligible sets) that the set $E:=\left\{z \in \mathbb{C}^{N} ; \psi(z)<\psi^{*}(z)\right\}$ is pluripolar. Therefore, in particular, the set $E$ is of $2 N$-dimensional Lebesgue measure zero.

Suppose Theorem 3 is not true for some $N>1$. Then there is a function $g$ holomorphic in a ball $B\left(z_{o}, R\right)$ with $z_{o} \in \mathcal{D}, R>r:=\operatorname{dist}\left(z_{o}, \partial \mathcal{D}\right)$ such that $g(z)=f(z)$ for $z \in B\left(z_{o}, r\right)$.

Let $b_{o}$ be a fixed point of $\partial \mathcal{D}$ such that $\left\|b_{o}-z_{o}\right\|=r$.

Since the ball $B\left(z_{o}, r\right)$ is non-thin at the point $b_{o}$,we have

$$
\limsup _{z \rightarrow b_{o}, z \in B\left(z_{o}, r\right)} \psi^{*}(z)=\psi^{*}\left(b_{o}\right) \text {. }
$$

Therefore, there is a sequence $\left\{z_{k}^{\prime}\right\} \subset B\left(z_{o}, r\right)$ such that $z_{k}^{\prime} \rightarrow b_{o}$, and $\psi^{*}\left(z_{k}^{\prime}\right) \rightarrow \psi^{*}\left(b_{o}\right)$ as $k \rightarrow \infty$. It follows that $\psi^{*}\left(b_{o}\right) \leq 1$. Since $b_{o} \in \partial D$, we have $\psi^{*}\left(b_{o}\right) \geq 1$. Therefore, $\psi^{*}\left(b_{o}\right)=1$.

We know that the $2 N$-dimensional Lebesgue measure $v_{2 N}(E)=0$. Therefore, by the sub-mean-value property, for every $k \geq 1$ there is a point $z_{k} \in B\left(z_{k}^{\prime}, \frac{1}{k}\right) \cap B\left(z_{o}, r\right) \backslash E$ such that $\psi\left(z_{k}\right)=\psi^{*}\left(z_{k}\right),\left|\psi^{*}\left(z_{k}^{\prime}\right)-\psi\left(z_{k}\right)\right|<\frac{1}{k}$. It is clear that the sequence $\left\{z_{k}\right\}$ satisfies the following properties:

$$
z_{k} \in B\left(z_{o}, r\right), z_{k} \rightarrow b_{o}, \psi\left(z_{k}\right)=\psi^{*}\left(z_{k}\right), \psi\left(z_{k}\right) \rightarrow \psi^{*}\left(b_{o}\right) .
$$


Put $b_{k}=z_{k} / \psi\left(z_{k}\right)(k \geq 1)$. Then $\psi\left(b_{k}\right)=\psi^{*}\left(b_{k}\right)=1$, in particular, $b_{k} \in \partial \mathcal{D}$ for $k \geq 1$, and $b_{k} \rightarrow b_{o}$ as $k \rightarrow \infty$.

Fix $k$ so large that $b:=b_{k} \in B\left(z_{o}, R\right)$. Put

$$
\begin{aligned}
G_{r} & :=\left\{\lambda \in \mathbb{C} ; \lambda b \in B\left(z_{o}, r\right)\right\}, \\
G_{R} & :=\left\{\lambda \in \mathbb{C} ; \lambda b \in B\left(z_{o}, R\right)\right\} .
\end{aligned}
$$

One can easily check that the sets $G_{r}, G_{R}$ are open, convex, nonempty (because $\lambda_{o} b \in G_{r}$ for $\lambda_{o}:=\psi\left(z_{k}\right)$, and $\left.G_{r} \subset G_{R}\right)$. Moreover, $G_{r} \subset \Delta:=$ $\{|\lambda|<1\}$, and $1 \in G_{R}$.

The function $f(\lambda b)$ (resp., $g(\lambda b)$ ) is holomorphic in $\Delta$ (resp., in $G_{R}$ ), and $f(\lambda b)=g(\lambda b)$ for $\lambda \in G_{r}$. Therefore, $f(\lambda b)=g(\lambda b)$ on $\Delta \cap G_{R}$. It follows that $g(\lambda b)$ is an analytic continuation of $f(\lambda b)$ across $\lambda=1$, contrary to the Fabry Theorem for $N=1$. We have got a contradiction showing that Theorem 3 is true.

Remark. The present proof of Theorem 3 - with no assumption on the continuity of the function $\psi^{*}$ - is a joint result of the author and Professor Azimbay Sadullaev.

3. Fatou-Hurwitz-Polya Theorem. First we shall state Fatou-Hurwitz -Polya Theorem for a series of homogeneous polynomials of $N$ complex variables.

Theorem 4. Let $f$ be a function holomorphic in a neighborhood of $0 \in \mathbb{C}^{N}$. Let

$$
f(z)=\sum_{0}^{\infty} Q_{j}(z), \quad Q_{j}(z)=\sum_{|\alpha|=j} \frac{f^{(\alpha)}(0)}{\alpha !} z^{\alpha},
$$

be its Taylor series development around 0 . Then there exists a sequence $\epsilon=\left\{\epsilon_{j}\right\}$ with $\epsilon_{j} \in\{-1,1\}$ (resp., $\epsilon_{j} \in\{0,1\}$ ) such that the function

$$
f_{\epsilon}(z):=\sum_{j=0}^{\infty} \epsilon_{j} Q_{j}(z), \quad z \in \mathcal{D},
$$

has no analytic continuation across any boundary point of the domain of convergence $\mathcal{D}:=\left\{\psi^{*}(z)<1\right\}$ of series (3.0), where

$$
\psi(z):=\limsup _{j \rightarrow \infty} \sqrt[j]{\left|Q_{j}(z)\right|} .
$$

For $N=1$ this theorem (with $\epsilon_{j} \in\{-1,1\}$ ) is due to Fatou-HurwitzPolya (Theorem 4.2.8 in [1]).

Now, we shall present an $N$-dimensional version of the Fatou-HurwitzPolya theorem for $N$-tuple power series

$$
f(z)=\sum_{|\alpha| \geq 0} c_{\alpha} z^{\alpha}
$$


where $c_{\alpha} z^{\alpha}$ is a monomial of $N$ complex variables $z=\left(z_{1}, \ldots, z_{N}\right)$ of degree $|\alpha|:=\alpha_{1}+\cdots+\alpha_{N}$. The set $\mathcal{D}:=\left\{a \in \mathbb{C}^{N}\right.$; the series (3.1) is absolutely convergent in a neighborhood of $a\}$ is called a domain of convergence of the multiple power series (3.1).

It is known that $\mathcal{D}=\left\{z \in \mathbb{C}^{N} ; h(z)<1\right\}$ is a complete $N$-circular (hence, in particular, $\mathcal{D}$ is balanced) domain whose Minkowski's functional $h \equiv h_{\mathcal{D}}$ is given by the formula $h(z)=M^{*}(z)$, where

$$
\begin{aligned}
M(z) & :=\limsup _{|\alpha| \rightarrow \infty} \sqrt[|\alpha|]{\left|c_{\alpha} z^{\alpha}\right|} \\
& =\limsup _{k \rightarrow \infty} \max \left\{\sqrt[|\alpha|]{\left|c_{\alpha} z^{\alpha}\right|} ;|\alpha|=k\right\}, \quad z \in \mathbb{C}^{N} .
\end{aligned}
$$

Moreover, $h\left(z_{1}, \ldots, z_{N}\right)=h\left(\left|z_{1}\right|, \ldots,\left|z_{N}\right|\right)$ for all $z \in \mathbb{C}^{N}$, and $h$ is continuous (see [2], Lemma 1.7.1 (b)).

Theorem 5. If the domain of convergence $\mathcal{D}$ of (3.1) is not empty, then there exists a multiple sequence $\epsilon=\left\{\epsilon_{\alpha}\right\}$ with $\epsilon_{\alpha} \in\{-1,1\}$ (resp., with $\left.\epsilon_{\alpha} \in\{0,1\}\right)$ such that the function

$$
f_{\epsilon}(z):=\sum_{|\alpha| \geq 0} \epsilon_{\alpha} c_{\alpha} z^{\alpha}, \quad z \in \mathcal{D}
$$

has no analytic continuation across any boundary point of $\mathcal{D}$.

We shall see that Theorems 4 and 5 are direct consequences of the following Lemma 3.2.

Let $\mathcal{X}:=\{0,1\}^{\mathbb{N}}\left(\right.$ resp. $\left.\{-1,1\}^{\mathbb{N}}\right)$ be the space of all sequences $x=$ $\left(x_{1}, x_{2}, \ldots\right)$ where $x_{j}=0$, or $x_{j}=1$ (resp. $x_{j}=-1$, or $x_{j}=1$ ) for $j=1,2, \ldots$ Endow $\mathcal{X}$ in the topology determined by the metric

$$
\rho(x, y):=\sum_{j=1}^{\infty} \frac{1}{2^{j}} \frac{|x-y|_{j}}{1+|x-y|_{j}},
$$

where

$$
|x-y|_{j}:=\max \left\{\left|x_{k}-y_{k}\right| ; k=1, \ldots, j\right\} .
$$

One can easily check that $\mathcal{X}$ is a complete metric space, and therefore, it has Baire property.

Moreover, in the topology a sequence $\{x(n)\}$ of elements of $\mathcal{X}$ converges to an element $x \in \mathcal{X}$ if and only if for every $k_{o} \in \mathbb{N}$ there exists $n_{o} \in \mathbb{N}$ such that $x_{k}(n)=x_{k}$ for $k=1, \ldots, k_{o}, n \geq n_{o}$.

Remark 3.1. Let $\left\{f_{k}\right\}$ be a sequence of holomorphic functions in an open subset $\Omega$ of $\mathbb{C}^{n}$. Then the following three conditions are equivalent:

(1) the series $\sum_{1}^{\infty}\left|f_{k}(z)\right|$ converges at each point $z \in \Omega$, and its sum $\varphi(z):=\sum_{1}^{\infty}\left|f_{k}(z)\right|$ is locally bounded on $\Omega$; 
(2) the series $\sum_{1}^{\infty} f_{k}$ converges locally normally in $\Omega$, i.e. for every point $a$ of $\Omega$ there exists a neighborhood $U$ of $a$ such that the series $\sum_{1}^{\infty}\left\|f_{k}\right\|_{U}$ is convergent;

(3) the series $\sum_{1}^{\infty}\left|f_{k}\right|$ converges locally uniformly in $\Omega$.

Proof. It is clear that $(2) \Rightarrow(3) \Rightarrow(1)$.

Suppose now (1) is true, and let $E(a, r):=\left\{z \in \mathbb{C}^{n} ;\left|z_{j}-a_{j}\right|<r(j=\right.$ $1, \ldots, n)\}$ be a polydisk whose closure is contained in $\Omega$. Then there is a positive constant $M$ such that $\sum_{1}^{\infty}\left|f_{k}(z)\right| \leq M$ for all $z \in E(a, r)$. By the Cauchy integral formula

$\left|f_{k}(z)\right| \leq \mu_{k}:=\left(\frac{1}{\pi r}\right)^{n} \int_{0}^{2 \pi} \ldots \int_{0}^{2 \pi}\left|f_{k}\left(a_{1}+r e^{i t_{1}}, \ldots, a_{n}+r e^{i t_{n}}\right)\right| d t_{1} \ldots d t_{n}$,

for all $z \in E\left(a, \frac{r}{2}\right)$ and $k \geq 1$.

By Lebesgue monotonous convergence theorem the series $\sum_{1}^{\infty} \mu_{k}$ is convergent, and so is the series $\sum_{1}^{\infty}\left\|f_{k}\right\|_{U}$ with $U:=E\left(a, \frac{r}{2}\right)$.

We shall see that our extensions of the classical Fatou-Hurwitz-Polya Theorem (Theorem 4.2.8 in [1]) are a direct consequence of the following Lemma 3.2 (slight modification of Lemma 5, p. 97 in [5]).

Lemma 3.2. Let $\mathcal{X}$ denote any of the two metric spaces $\{0,1\}^{\mathbb{N}}$ or $\{-1,1\}^{\mathbb{N}}$. Let $\left\{f_{k}\right\}$ be a sequence of holomorphic functions in an open neighborhood $\Omega$ of the closure of a ball $B=B(w, r)$ such that the series $\sum_{1}^{\infty}\left|f_{k}(z)\right|$ converges at every point $z \in B$. Let a be a boundary point of $B$.

Then, either the series $\sum_{1}^{\infty} f_{k}$ is normally convergent on a neighborhood of $a$, or there exists a subset $\mathcal{R}$ of $\mathcal{X}$ of the first category such that for every $x \in \mathcal{X} \backslash \mathcal{R}$ the function $f_{x}(z):=\sum_{k} x_{k} f_{k}(z), z \in B$, has a singular point at a (in other words, $f_{x}$ cannot be analytically continued to any neighborhood of a).

Proof. Given a natural number $m$, let $\mathcal{R}_{m}$ denote the set of all $x \in \mathcal{X}$ such that there exists a holomorphic function $\tilde{f}_{x}$ on $E_{m}$ (where $E_{m}$ is the polydisk $E_{m}:=E\left(a, \frac{1}{m}\right)$ with center $a$ and radius $\left.\frac{1}{m}\right)$ such that $\left|\tilde{f}_{x}(z)\right| \leq m$ on the polydisk, and $\tilde{f}_{x}(z)=f_{x}(z)$ for all $z \in B \cap E_{m}$. By definition, we put $\mathcal{R}_{m}=\emptyset$, if $m<1 / \operatorname{dist}(a, \partial \Omega)$.

It is clear that the set $\mathcal{R}:=\bigcup_{1}^{\infty} \mathcal{R}_{m} \equiv\left\{x \in \mathcal{X} ; f_{x}\right.$ has an analytic continuation across $a$ \}.

The lemma will be proved if we show that the following two claims are true.

Claim 1. The set $\mathcal{R}_{m}$ is closed in the space $\mathcal{X}$.

Claim 2. If the interior of $\mathcal{R}_{m}$ is not empty, then the series $\sum_{1}^{\infty} f_{k}$ is normally convergent on a neighborhood of a. 
Indeed, if the series $f_{x}:=\sum_{1}^{\infty} x_{k} f_{k}$ converges normally on no neighborhood $U$ of $a$, then for every $m \geq 1$ the set $\mathcal{R}_{m}$ is closed and has empty interior. Hence, the set $\mathcal{R}:=\bigcup_{1}^{\infty} \mathcal{R}_{m} \equiv\left\{x \in \mathcal{X} ; f_{x}\right.$ has an analytic continuation $\tilde{f}_{x}$ across $\left.a\right\}$ is of the first category, and for every $x \in \mathcal{X} \backslash \mathcal{R}$ the function $f_{x}$ has a singular point at $a$, i.e. $f_{x}$ has no analytic continuation across $a$. We say that a function $\tilde{f}_{x}$ holomorphic on a polydisk $E$ with center $a$ is an analytic continuation of $f_{x}$ across $a$, if $\tilde{f}_{x}(z)=f_{x}(z)$ on $B \cap E$.

Proof of Claim 1. Let $\{x(j)\}$ be a sequence of elements of $\mathcal{R}_{m}$ convergent to $x \in \mathcal{X}$. Let $\left\{h_{j}\right\} \equiv\left\{\tilde{f}_{x(j)}\right\}$ be a sequence of holomorphic functions on $E_{m}$ such that $\left|h_{j}(z)\right| \leq m$ on $E_{m}$ and $h_{j}(z)=f_{x(j)}(z)$ on the intersection $B \cap E_{m}$ for $j \geq 1$. Observe that for every $k_{o}$ there exists $j_{o}$ such that $\left|f_{x(j)}(z)-f_{x}(z)\right| \leq \sum_{k>k_{o}} 2\left|f_{k}(z)\right|$ for all $z \in B \cap E_{m}$ and for all $j>j_{o}$. It follows that the sequence $\left\{h_{j}\right\}$ is convergent at each point of $B \cap E_{m}$. By Vitali's theorem the sequence $\left\{h_{j}\right\}$ is locally uniformly convergent on $E_{m}$ to a holomorphic function $h$ bounded by $m$ and identical with $f_{x}$ on $E_{m} \cap B$, which shows that $x \in \mathcal{R}_{m}$.

Proof of Claim 2. If $\mathcal{R}_{m}$ has a nonempty interior, then there exist $x(0)=\left(x_{1}(0), x_{2}(0), \ldots\right) \in \mathcal{R}_{m}$ and a natural number $k_{o}$ such that

$$
x \in \mathcal{X}, x_{j}=x_{j}(0) \quad\left(j=1, \ldots, k_{o}\right) \quad \Longrightarrow \quad x \in \mathcal{R}_{m} .
$$

Put

$$
M:=\sup \left\{\sum_{k=1}^{k_{0}}\left|f_{k}(z)\right| ; z \in E_{m}\right\}, \quad u_{k}:=\Re f_{k}, \quad v_{k}:=\Im f_{k} .
$$

By implication $(2) \Rightarrow(3)$ of Remark 3.1 it is sufficient to show that

$$
\sum_{k=1}^{\infty}\left|f_{k}(z)\right| \leq M+4 m, \quad z \in E_{m}
$$

Let $A$ be a finite subset of $\mathbb{N} \backslash\left[1, k_{0}\right]$. Given a fixed point $z$ of $E_{m}$, put

$$
A_{1}:=\left\{k \in A ; u_{k}(z) \geq 0\right\}, \quad A_{2}:=\left\{k \in A ; u_{k}(z)<0\right\} .
$$

It is clear that $A=A_{1} \cup A_{2}, A_{1} \cap A_{2}=\emptyset$. Consider two cases.

Case 1: $\mathcal{X}=\{0,1\}^{\mathbb{N}}$. Let $x(j)=\left(x_{1}(j), x_{2}(j), \ldots\right)(j=1,2)$ be two points of the interior of $\mathcal{R}_{m}$ defined by the formulas:

$$
\begin{aligned}
& x_{k}(j)=x_{k}(0), \quad k=1, \ldots, k_{0}, \quad j=1,2 ; \\
& x_{k}(j)=x_{k}(0), \quad k>k_{0}, \quad k \notin A, \quad j=1,2 ; \\
& x_{k}(1)=1, \quad x_{k}(2)=0, \quad k \in A_{1} ; \\
& x_{k}(1)=0, \quad x_{k}(2)=1, \quad k \in A_{2} .
\end{aligned}
$$


Then

$$
\sum_{k \in A}\left|u_{k}(z)\right| \leq\left|\sum_{k \in A}\left(x_{k}(1)-x_{k}(2)\right) f_{k}(z)\right|=\left|\tilde{f}_{x(1)}(z)-\tilde{f}_{x(2)}(z)\right| \leq 2 m .
$$

By the arbitrary property of $A$ and $z$ one gets

$$
\sum_{k=k_{0}+1}^{\infty}\left|u_{k}(z)\right| \leq 2 m, \quad z \in E_{m}
$$

The same argument gives

$$
\sum_{k=k_{0}+1}^{\infty}\left|v_{k}(z)\right| \leq 2 m, \quad z \in E_{m}
$$

Hence

$$
\sum_{k=1}^{\infty}\left|f_{k}(z)\right|=\left(\sum_{k=1}^{k_{0}}+\sum_{k=k_{0}+1}^{\infty}\right)\left|f_{k}(z)\right| \leq M+4 m, \quad z \in E_{m} .
$$

Case 2: $\mathcal{X}=\{-1,1\}^{\mathbb{N}}$. Now we define two elements $x(1), x(2)$ of the interior of $\mathcal{R}_{m}$ by the formulas:

$$
\begin{aligned}
& x_{k}(j)=x_{k}(0), \quad k=1, \ldots, k_{0}, \quad j=1,2 ; \\
& x_{k}(j)=x_{k}(0), \quad k>k_{0}, \quad k \notin A, \quad j=1,2 ; \\
& x_{k}(1)=1, \quad x_{k}(2)=-1, \quad k \in A_{1} ; \\
& x_{k}(1)=-1, \quad x_{k}(2)=1, \quad k \in A_{2} .
\end{aligned}
$$

Then

$$
2 \sum_{k \in A}\left|u_{k}(z)\right| \leq\left|\sum_{k \in A}\left(x_{k}(1)-x_{2}(2)\right) f_{k}(z)\right|=\left|\tilde{f}_{x(1)}(z)-\tilde{f}_{x_{k}(2)}(z)\right| \leq 2 m .
$$

Hence, by the analogous argument as in the proof of the case 1, we get

$$
\sum_{k=1}^{\infty}\left|f_{k}(z)\right| \leq M+4 m, \quad z \in E_{m},
$$

which ends the proof of the case 2 .

Corollary 3.3. Let $\left\{f_{k}\right\}$ be a sequence of holomorphic functions on an open set $\Omega \subset \mathbb{C}^{N}$. Let $D$ denote the set of all points a in $\Omega$ such that the series $\sum_{1}^{\infty} f_{k}$ is absolutely convergent at every point of a neighborhood of a. Assume that the sum $\varphi(z):=\sum_{1}^{\infty}\left|f_{k}(z)\right|$ is locally bounded in $D$, and $\bar{D} \subset \Omega$. Let $\mathcal{X}$ be any of the two metric spaces $\{0,1\}^{\mathbb{N}}$ or $\{-1,1\}^{\mathbb{N}}$.

Then there exists a subset $\mathcal{R}$ of $\mathcal{X}$ of the first category such that for every point $x \in \mathcal{X} \backslash \mathcal{R}$ the holomorphic function $f_{x}(z):=\sum_{k=1}^{\infty} x_{k} f_{k}(z), z \in D$, cannot be continued analytically across any boundary point of $D$. 
Proof. Let $\left\{w_{j}\right\}$ be the sequence of all rational points of $D$ (or any countable dense subset of $D)$. Let $a_{j}$ be a point of $\partial D$ such that $\left\|w_{j}-a_{j}\right\|=$ $\operatorname{dist}\left(w_{j}, \partial D\right)$. By Lemma 3.2 for every $j$ there exists a subset $\mathcal{R}_{j}$ of $\mathcal{X}$ of the first category such that for every $x \in \mathcal{X} \backslash \mathcal{R}_{j}$ the function $f_{x}$ has a singular point at $a_{j}$. The set $\mathcal{R}:=\bigcup \mathcal{R}_{j}$ is again of the first category such that for every $x \in \mathcal{X} \backslash \mathcal{R}$ the function $f_{x}$ has analytic extension across no boundary point of $D$.

Proof of Theorems 4 and 5. It is sufficient to apply Lemma 3.2 with $\Omega=\mathbb{C}^{N}$, with $f_{k}=Q_{k}$ and $f_{k}=c_{\alpha(k)} z^{\alpha(k)}\left(k \in \mathbb{Z}_{+}\right)$, respectively, where $\alpha: \mathbb{Z}_{+} \ni k \mapsto \alpha(k) \in \mathbb{Z}_{+}^{N}$ is a one-to-one mapping, and with $D$ replaced by the domain of convergence $\mathcal{D}$ of the corresponding power series.

Remark 3.4. The author would like to draw reader's attention to the fact that, unfortunately, the proofs of Theorems 4 and 5 published in [6] contain flaws.

\section{REFERENCES}

[1] Bieberbach, L., Analytische Fortsetzung, Springer-Verlag, Berlin 1955.

[2] Jarnicki, M., Jakóbczak, P., Wstęp do teorii funkcji holomorficznych wielu zmiennych zespolonych, Wydawnictwo Uniwersytetu Jagiellońskiego, Kraków, 2002.

[3] Klimek, M., Pluripotential Theory, Oxford University Press, New York, 1991.

[4] Luh, W., Universal approximation properties of convergent power series on open sets, Analysis 6 (1986), 191-207.

[5] Saint Raymond, J., Transformations séparément analytiques, Ann. Inst. Fourier (Grenoble) 40 (1990), 79-101.

[6] Siciak, J., Generalizations of a theorem of Fatou, Zeszyty Naukowe UJ 11 (1966), $81-84$.

[7] Siciak, J., Sets in $\mathbb{C}^{N}$ with vanishing global extremal function and polynomial approximation, Ann. Fac. Sci. Toulouse 20 (2011), no. S2, 189-209.

Józef Siciak

Institute of Mathematics

Jagiellonian University

Łojasiewicza 6

30-348 Kraków

Poland

e-mail: Jozef.Siciak@im.uj.edu.pl

Received May 16, 2011 